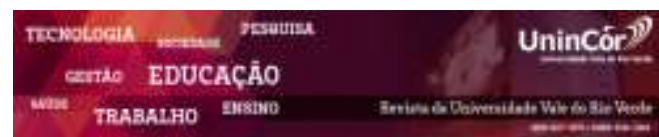

Revista da Universidade Vale do Rio Verde ISSN: 1517-0276 / EISSN: 2236-5362 v. 17 | n. 1 | Ano 2019

Bianca dos Santos Silva Faculdade Maria Milza - FAMAM biancasilva.e@gmail.com

Roberto Carlos de Carvalho Cerqueira Faculdade Maria Milza - FAMAM robertocarlosc13@hotmail.com

Larissa Rolim Borges-Paluch Faculdade Maria Milza - FAMAM larissapaluch@gmail.com

Cláudia Cecília Blaszkowski de Jacobi Faculdade Maria Milza - FAMAM cbjacobi@gmail.com

\section{PERFIL EPIDEMIOLÓGICO E SAÚDE BUCAL DE PACIENTES ATENDIDOS EM UMA CLÍNICA INTEGRADA DE ODONTOLOGIA}

\section{RESUMO}

Conhecer o perfil de pacientes que buscam atendimento em serviços de saúde é de fundamental importância para o desenvolvimento de um bom planejamento visando à promoção, prevenção e intervenção na saúde destes indivíduos. O objetivo do presente estudo foi conhecer o perfil epidemiológico de pacientes atendidos em uma clínica-escola de Odontologia. Trata-se de uma pesquisa epidemiológica transversal, descritiva, documental, de abordagem quantitativa. Para a coleta de dados foi criada uma ficha-registro para armazenamento das informações individuais referentes às variáveis sociodemográficas, comportamentais, e de saúde no período de Janeiro/2015 a Dezembro/2016. Os achados mostraram que os usuários são, predominantemente, do sexo feminino $(65,5 \%)$, melanoderma $(35,5 \%)$ e idade entre 30-49 anos $(55,5 \%)$. Sobre o histórico odontológico, $35,1 \%$ indicaram a dor de origem dentária como motivo para a consulta e em relação ao tipo de tratamentos $61,3 \%$ realizou raspagem periodontal. O estudo possibilitou reconhecer as características da população abrangida pela clínica-escola e identificar as suas demandas. Logo, espera-se contribuir para o planejamento da assistência prestada e o direcionamento das ações de intervenção em saúde.

Palavras-chave: Epidemiologia. Perfil de Saúde. Saúde bucal.

\section{EPIDEMIOLOGIC PROFILE AND DENTAL HEALTH OF PATIENTS IN A DENTAL SCHOOL CLINIC}

\begin{abstract}
To know the profile of patients who look for service in health services is of basic importance for the development of a good projection aiming for the promotion, prevention and intervention in the health of these individuals. The aim of this study was to assess the epidemiologic profile of patients in a dental school clinic. To reach our objective an epidemiologic, transversal, descriptive, documental, and quantitative research was carried out. Sociodemographic, behavioral and health information of individuals collected from January 2015 to December 2016 were stored in especially designed register files. Data
\end{abstract}


analysis showed that most of the patients were women $(65.5 \%)$, black $(35.5 \%)$, and aged 30 to 49 years old $(55.5 \%)$. With regard to dental case history, $35.1 \%$

stated that dental pain was the reason for the appointment, and in terms of treatment, $61.3 \%$ underwent periodontal scaling. This research enabled us to determine the characteristics and needs of the dental school patients and the results will contribute to the planning of assistance and health promotion actions.

Keywords: Epidemiology. Health Profile. Dental health.

Recebido em: 05/092018 - Aprovado em: 31/003/2019 - Disponibilizado em: 15/07/2019

\section{INTRODUÇÃO}

No âmbito da saúde pública, os serviços odontológicos foram incrementados no Sistema Único de Saúde (SUS) com a implantação do programa Brasil Sorridente, lançado pelo Ministério da Saúde (MS), em 2004, por meio da Política Nacional de Saúde Bucal. Essa política determina que sejam utilizados dados da Epidemiologia e sobre territórios para subsidiar o planejamento de programas de prevenção e tratamento (BRASIL, 2011).

Roncalli et al (2006) considera que no Brasil são tímidas as experiências do uso da Epidemiologia nos serviços de saúde bucal. O autor observa a inserção tardia do país no processo de construção de uma base de dados alimentada a partir das informações obtidas em estudos epidemiológicos. Os expressivos levantamentos em saúde bucal sob a condução do MS em 1996, 2003 e 2010 (BRASIL, 2011).

Os estudos relacionados ao perfil de usuários de serviços de saúde constituem ferramentas estratégicas para os gestores desses serviços. Além disso, os dados podem possibilitar a avaliação do padrão de atendimento dispensado ao usuário, além de detectar possíveis limitações de atuação (MAIA et al, 2016).

A prestação de serviços por instituições de ensino superior configura-se como uma atividade de extensão e representa um valioso instrumento fomentador de aprendizado (BOLAN; MOTTA, 2007). Por este cenário percorre a formação odontológica, cujo exercício técnico da profissão se desenvolve em ambulatório-escola. Nestes espaços são prestados atendimentos à comunidade, com ênfase no caráter tecnicista da Odontologia, embora a trajetória acadêmica envolva também a aquisição de conhecimentos biológicos, humanos e sociais (PEREIRA et al, 2011).

O estudo teve como objetivo traçar o perfil epidemiológico de pacientes atendidos em uma clínica-escola, visando subsidiar o planejamento de ações de saúde compatíveis com a demanda local. 


\section{METODOLOGIA}

Trata-se de uma pesquisa epidemiológica transversal, descritiva, documental, de abordagem qualitativa. A pesquisa teve como cenário uma clínica-escola pertencente a uma Faculdade de Odontologia sediada no município de Cruz das Almas, Bahia.

A clínica oferece serviços odontológicos nas especialidades de dentística, odontopediatria, endodontia, periodontia, prótese, cirurgia e radiologia. No ano de 2016 foram realizados cerca de 4.370 procedimentos em 1.070 pacientes (adultos e crianças) atendidos.

As informações para a pesquisa foram extraídas de prontuários clínicos pertencentes a pacientes que realizaram tratamento odontológico na clínica-escola entre janeiro/2015 e dezembro/2016. O critério de inclusão foi idade acima 18 anos e de exclusão foi grafia ilegível e tratamento odontológico em andamento.

Os dados utilizados foram referentes às variáveis: sociodemográficas, comportamentais, histórico médico e odontológico dos pacientes. As informações são apresentados por meio de frequências absoluta e relativa, além do cálculo de média aritmética simples nas tabelas e figura.

O presente projeto possui aprovação do Comitê de Ética em Pesquisa e atentou a todos os princípios éticos contidos na Resolução n 466/12 do Conselho Nacional de Saúde.

\section{RESULTADOS E DISCUSSÃO}

Variável Sociodemográfica

Em relação aos aspectos sociodemográficos, das 194 fichas avaliadas, verificou-se que cerca de $70 \%$ dos pacientes atendidos pertencem ao sexo feminino e $68,0 \%$ se autodeclaram $^{1}$ melanoderma ou faioderma. Quanto à faixa etária, observou-se predomínio da população com idade entre 30 e 49 anos $(55,5 \%)$, a maioria declarou não possuir companheiro $(58,3 \%)$ e $65,9 \%$ exerce atividade remunerada.

A população majoritariamente feminina também foi verificada em outros estudos de mesma natureza como de Sanchez e Drumond (2011) e França et al (2011).

Gomes, Nascimento e Araújo (2007) atrelam este fato ao papel social atribuído a homens e mulheres. Pois para o imaginário da sociedade, o ato de cuidar é próprio do universo feminino, enquanto os homens são associados à invulnerabilidade, força e virilidade. Outras questões, como o medo da descoberta de uma doença grave e a vergonha da exposição do corpo perante o profissional de saúde, também contribuem para a pouca procura dos homens ao serviço de assistência.

A raça/cor verificada nos prontuários, reflete a composição étnica predominante no estado da Bahia é composta majoritariamente $(79,3 \%)$ por melanodermas e faiodermas (IBGE, 2016a).

\footnotetext{
${ }^{1}$ A nomenclatura adotada para a identificação da raça/cor da pele na área médico-legal foi introduzida por Roquette-Pinto em 1933, sendo melanoderma (melanodermos) designado para pretos; leucoderma (leucodermos) para brancos; faioderma (phaiodermos) para pardos; xantoderma (xanthodermos) para amarelo e eritroderma para indígena.
} 
Em relação à variável faixa-etária, Gibilini et al (2010) avaliaram o acesso a serviços odontológicos e autopercepção da saúde bucal em adolescentes, adultos e idosos e, ao compará-los, concluíram que o último grupo é o que menos procura o dentista. Esses autores verificaram que para 58,5\% dos idosos a última visita odontológica ocorrera em um período superior há três anos, enquanto que para $60,1 \%$ dos adolescentes e $47,9 \%$ dos adultos a última consulta fora realizada há menos de um ano.
Em estudo realizado por Gabardo et al (2015) investigando a associação entre as variáveis sociodemográficas, socioeconômicas, psicossociais e comportamentais e a saúde bucal, foi possível concluir que a autopercepção individual de saúde bucal mais desfavorável foi relatada por mulheres, por pessoas com idade mais avançada, por indivíduos com baixa renda familiar e menor escolaridade.

Tabela 1. Frequências absoluta (n) e relativa (\%) referentes aos dados sociodemográficos dos pacientes $(\mathrm{N}=194)$. Clínica-escola de Odontologia, Cruz das Almas (BA). 2017.

\begin{tabular}{|c|c|c|c|}
\hline VARIÁVEIS & $\mathbf{N}(\%)$ & VARIÁVEIS & $\mathbf{N}(\%)$ \\
\hline SEXO & & \multicolumn{2}{|c|}{ RAÇA/COR (AUTORREFERIDA) } \\
\hline Feminino & $127(65,5)$ & Melanoderma & $69(35,5)$ \\
\hline Masculino & $67(34,5)$ & Faioderma & $63(32,5)$ \\
\hline OCUPAÇÃO & & Leucoderma & $17(8,8)$ \\
\hline Remunerada & $128(65,9)$ & NI & $45(23,2)$ \\
\hline Não remunerada & $27(14,0)$ & \multicolumn{2}{|l|}{ FAIXA ETÁRIA } \\
\hline NI & $39(20,1)$ & 18 a 29 & $44(23,0)$ \\
\hline ESTADO CIVIL & & 30 a 39 & $62(31,9)$ \\
\hline Sem companheiro & $113(58,3)$ & 40 a 49 & $46(23,6)$ \\
\hline Com companheiro & $73(37,6)$ & 50 a 59 & $24(12,0)$ \\
\hline \multirow[t]{2}{*}{$\mathrm{NI}$} & $08(4,1)$ & $>60$ & $16(8,5)$ \\
\hline & & NI & $02(1,0)$ \\
\hline
\end{tabular}

Legenda: NI: não informado

\section{Variáveis Comportamentais e Histórico Médico}

Os hábitos comportamentais registrados nos prontuários revelaram que $55,6 \%$ dos pacientes não consomem bebida alcoólica e 9,8\% são ou foram fumantes. O consumo de dieta cariogênica foi relatado por $39,7 \%$ dos pacientes e
$58,0 \%$ afirmam que a consulta odontológica mais recente ocorreu há cerca de um ano (Tabela 2).

Com relação ao histórico médico destes pacientes, 63,7\% relataram não possuir alterações sistêmica. Dentre os usuários que possuem doenças, as mais prevalentes foram a ansiedade e a hipertensão arterial, afetando 15,0\% e 11,0\%, respectivamente (Tabela 3 ). 
Tabela 2. Frequências absoluta (n) e relativa (\%) referentes aos dados comportamentais dos pacientes $(\mathrm{N}=194)$. Clínica-escola de Odontologia, Cruz das Almas (BA). 2017.

\begin{tabular}{|c|c|c|c|}
\hline \multirow{2}{*}{\multicolumn{2}{|c|}{$\begin{array}{l}\text { VARIÁVEIS } \mathbf{N}(\%) \\
\text { HÁBITO ETILISTA ATUAL OU PASSADO }\end{array}$}} & VARIÁ VEIS & \multirow[t]{2}{*}{$\mathbf{N}(\%)$} \\
\hline & & DIETA CARIOGÊNICA & \\
\hline Presente & $81(41,8)$ & Sim & $77(39,7)$ \\
\hline Ausente & $108(55,6)$ & Não & $108(55,7)$ \\
\hline Sem informação & $05(2,6)$ & Sem informação & $09(4,6)$ \\
\hline \multicolumn{2}{|c|}{ HÁBITO TABAGISTA ATUAL OU PASSADO } & \multicolumn{2}{|c|}{ ÚLTIMA CONSULTA ODONTOLÓGICA } \\
\hline Ausente & $171(88,1)$ & Há menos de 1 ano & $113(58,0)$ \\
\hline Presente & $19(9,8)$ & Há mais de 1 ano & $81(37,0)$ \\
\hline NI & $04(2,1)$ & NI & $10(5,0)$ \\
\hline
\end{tabular}

Legenda: NI: não informado

Tabela 3 Frequências absoluta (n) e relativa (\%) de algumas das alterações sistêmicas presentes entre os pacientes $(\mathrm{N}=$ 194). Clínica-escola de Odontologia, Cruz das Almas (BA). 2017.

\begin{tabular}{lc|cr}
\multicolumn{1}{l|}{ VARIÁVEIS } & \multicolumn{1}{c}{ VARIÁVEIS } & \multicolumn{1}{c}{ N $(\%)$} \\
\hline \multicolumn{1}{l|}{ HIPERTENSÃO ARTERIAL SISTEMICA } & ANSIEDADE & \\
Sim & $22(11,3)$ & Sim & $165(85,0)$ \\
Não & $172(88,7)$ & Não \\
DEPRESSÃO & & DIABETES & \\
Sim & $07(3,6)$ & Sim & $03(1,6)$ \\
Não & $187(96,4)$ & Não & $191(98,4)$ \\
\hline
\end{tabular}

A verificação de hábitos comportamentais pesquisada por Andriola et al (2015), em seu estudo com pacientes odontológicos no Rio Grande do Sul, também constatou o predomínio de usuários que declararam não utilizar fumo ou bebidas alcoólicas.

Em relação a variáveis da história médica, neste estudo constatou-se que a alteração sistêmica mais relatada pelos usuários foi a ansiedade, seguida da hipertensão arterial sistêmica (HAS). Castillo et al (2000) definem a ansiedade como "um sentimento vago e desagradável de medo, apreensão, caracterizado por tensão ou desconforto derivado de antecipação de perigo, de algo desconhecido ou estranho". As repercussões deste estado alcançam à saúde física e contribuem para o surgimento de processos patológicos de interesse odontológico, como a desordem temporo-mandibular (DTM). A DTM é de natureza multifatorial, sendo que os motivos desencadeantes podem ter origem física e/ou psicológica. A correlação significativa entre a presença da desordem e a ansiedade e depressão também foi constatada por Silva et al (2014).

A HAS, referida neste estudo por $11,3 \%$ dos pacientes, é uma alteração caracterizada pela pressão sistólica maior ou igual a $140 \mathrm{mmHg}$ e a diastólica maior ou igual a $90 \mathrm{mmHg}$. A HAS representa um fator de risco para o desenvolvimento de doença cardiovascular e, segundo a literatura, acomete entre 10 a $20 \%$ da população adulta e $90 \%$ dos idosos. 
Para os portadores desse agravo é imperativo a aferição da pressão arterial prévia à intervenção odontológica, bem como a promoção do controle da ansiedade e redução do estresse a fim de minimizar a influencia do fator emocional na elevação da pressão arterial. As implicações desta condição para a Odontologia referem-se, sobretudo, aos cuidados quanto à administração de anestésicos e prescrição de fármacos antiinflamatórios, cujo mecanismo de ação pode interferir na medicação anti-hipertensiva (SPEZZIA; CALVOSO JUNIOR, 2017).

\section{Variável Histórico Odontológico}

As informações referentes à história odontológica apontam que a 'dor de origem dentária' foi o principal motivo para a busca pelo serviço para 35,1\% dos pacientes. Outras queixas, como a presença de cárie e sensibilidade, também foram citadas, respectivamente, $24,7 \%$ e $14,9 \%$ dos usuários.

Os dados revelaram também que durante a consulta inicial houve o diagnóstico de cárie ativa em $82,0 \%$ dos pacientes; e o registro periodontal simplificado (RPS) de maior prevalência correspondeu ao de número $2(25,3 \%)$. Dentre os principais tratamentos odontológicos realizados, a raspagem periodontal $(61,3 \%)$ foi o mais efetuado, seguido dos procedimentos restauradores $(59,3 \%)$ e a profilaxia $(43,3 \%)$ (Tabela 6).

Após análise do odontograma foi possível verificar que a faixa etária de 18-29 anos possui a maior quantidade média de dentes presentes, com 28 unidades; quanto à média de dentes cariados, 0 maior valor, que correspondeu a 7 , foi observado no grupo etário de 40-49 anos (Figura 1).

Tabela 6. Frequências absoluta (n) e relativa (\%) referentes aos dados de saúde relacionados à história odontológica. Clínica-escola de Odontologia, Cruz das Almas (BA). 2017.

\begin{tabular}{|c|c|c|c|}
\hline \multicolumn{2}{|c|}{ VARIÁVEIS N $(\%)$} & \multicolumn{2}{|c|}{ VARIÁVEIS $\quad$ N (\%) } \\
\hline \multicolumn{2}{|c|}{ MOTIVAÇÃO PARA CONSULTA* } & \multicolumn{2}{|c|}{ TRATAMENTO REALIZADO* } \\
\hline Dor & $68(35,1)$ & Raspagem & $119(61,3)$ \\
\hline Cárie & $48(24,7)$ & Restauração & $115(59,3)$ \\
\hline Sensibilidade & $29(14,9)$ & Exodontia & $39(20,1)$ \\
\hline Assintomático & $17(8,8)$ & Endodontia & $34(17,1)$ \\
\hline Tártaro & $17(8,8)$ & Aplicação de flúor & $41(21,1)$ \\
\hline Sangramento gengival & $06(3,1)$ & Profilaxia & $84(43,3)$ \\
\hline Insatisfação com a estética & $26(13,4)$ & Prótese total & $05(2,6)$ \\
\hline Reabilitação com prótese & $11(5,7)$ & Prótese parcial & $10(5,2)$ \\
\hline Restauração insatisfatória & $05(2,6)$ & Outros ou NI & $15(7,7)$ \\
\hline Outras ou NI** & $21(10,9)$ & \multicolumn{2}{|c|}{ REGISTRO PERIODONTAL SIMPLIFICADO } \\
\hline CÁRIE ATIVA & & Zero & $25(12,9)$ \\
\hline Sim & $159(82,0)$ & $\mathrm{Um}$ & $21(10,8)$ \\
\hline Não & $15(7,7)$ & Dois & $49(25,3)$ \\
\hline NI & $20(10,3)$ & Três & $36(18,6)$ \\
\hline \multicolumn{2}{|c|}{ ORIENTAÇÃO DE HIGIENE BUCAL } & Quatro & $21(10,8)$ \\
\hline
\end{tabular}




\begin{tabular}{lr|l|l} 
Sim & $72(37,1)$ & NI & $42(21,6)$ \\
Não & $111(57,2)$ & & \\
NI & $11(5,7)$ & & \\
\hline
\end{tabular}

Nota: *todos os tratamentos feitos em um mesmo paciente. Legenda: NI**: não informado

Figura 1. Média aritmética simples de dentes presentes e cárie ativa, segundo a faixa etária. Cruz das Almas (BA). 2017.

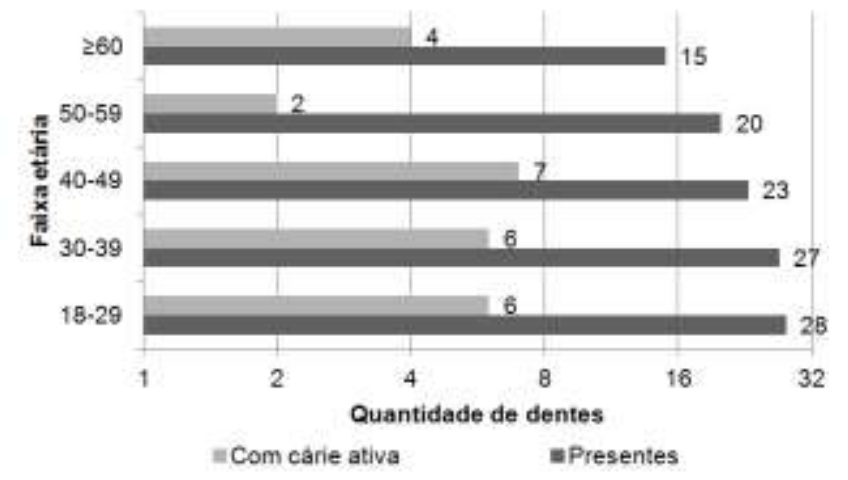

$\mathrm{Na}$ esfera coletiva, a análise de aspectos odontológicos feita por Nascimento et al (2013) concluiu que a saúde bucal dos brasileiros sofreu uma leve melhora; esta conclusão foi embasada na análise de dados provenientes dos levantamentos epidemiológicos realizados pelo MS. Os autores citam que este fato é consequência de ações como a adição de flúor à água e no dentifrício, assim como o incremento dos serviços restauradores e de políticas públicas. Nico et al (2016), no entanto, ressaltam que entre a população brasileira os agravos à saúde bucal ainda são prevalentes e representam um dos principais problemas da saúde pública.

Em relação da queixa de dor de origem dentária Domingos, Rossato e Bellini (2014) em seu estudo também constataram este problema como o principal motivo para consulta.

E Ferreira et al (2012) verificaram que a dor de dente representou a causa da consulta odontológica entre $33,44 \%$ dos investigados em seu estudo. Esses autores relatam ainda que a odontalgia associa-se à baixa renda, ao maior número de pessoas residentes no mesmo domicílio, à baixa frequência de escovação diária, ao alto intervalo entre as consultas odontológicas, ao maior período de tempo em que se deu a última consulta odontológica, à ansiedade odontológica, ao consumo de alimentos cariogênicos, à alta experiência de cárie e à presença de lesão cariosa não tratada.

Costa, Vasconcelos e Abreu (2013) analisaram a relação entre a qualidade de vida e a saúde bucal de adultos (35-44 anos) e concluíram que a presença da doença cárie associa-se a uma autopercepção negativa da qualidade de vida.

Em relação ao RPS Oliveira et al (2015) destacam que o valor 2 é atribuído quando, há presença de retentores de placa bacteriana, tais como cálculo, supra e/ou subgengival, e/ou margens restauradoras mal adaptadas. Segundo estes autores, a instrução de higiene, a remoção de placa subgengival e cálculo através de raspagem e correção de margens restauradoras são os tratamentos requeridos para solucionar a situação clínica.

Melo et al (2016) avaliaram o perfil periodontal de pacientes atendidos em uma faculdade, e também constataram predomínio deste escore, verificando-o em 55\% dos pacientes.

A média de dentes presentes em adultos foi de 25 unidades nesse estudo, e a maioria dos 
indivíduos $(57,2 \%)$ relatou nunca ter recebido orientação profissional de higiene bucal visando a prevenção dos agravos à saúde oral. Dados de um levantamento epidemiológico realizado pelo MS, entre 2002 e 2003, confirmam a necessidade das intervenções odontológicas, devidas, entre outros fatores, à precocidade das perdas dentárias, recomendando também o uso de prótese dentária em adolescentes de 15 a 19 anos de idade (BRASIL, 2004).

Resultado similar foi relatado por Gibilini et al (2010), que observaram que $64,3 \%$ dos adultos possuíam 20 ou mais unidades dentárias, enquanto que nos idosos a prevalência de edentulismo foi de 59,9\%. Verificaram, ainda, que maioria dos pesquisados haviam recebido informações de higiene bucal. Ao relacionar os resultados desses autores com os achados da presente pesquisa, conclui-se que há semelhança no que se refere à média de dentes presentes e oposição quanto ao nível de instrução sobre a saúde oral recebida.

Borges et al (2014) afirmam que a alta escolaridade é um fator de proteção contra a perda dentária. Seu estudo, realizado com residentes nos municípios do entorno de Belo Horizonte, Minas Gerais, traçou um perfil das perdas dentárias segundo o capital social, as características demográficas e socioeconômicas em adultos. Os resultados demonstraram que o grupo de baixa renda, analfabetos ou que tinham cursado somente o ensino fundamental perderam mais que quatro dentes quando comparados aos de renda mais alta e com maior escolaridade. Os autores apontam que a renda per capita e o nível de instrução dos adultos se associam às perdas dentárias.
De acordo com Cimoes et al (2007), a cárie ainda é o principal responsável pelas perdas dentárias, e a condição socioeconômica do indivíduo influencia a sua saúde bucal. Na infância, ela é uma doença comum que afeta de $60 \%$ a $90 \%$ das crianças em idade escolar nos países industrializados.

Fonseca, Nehmy e Mota (2015) relatam que a má condição da dentição, além de comprometer a mastigação e a fonação, produz constrangimento devido aos prejuízos estéticos. Os autores também acreditam que a manutenção de uma boca saudável é importante para a aquisição do bem-estar social, para a saúde do corpo e da mente, uma vez que os dentes desempenham funções na cavidade bucal e também possuem importância social.

\section{CONSIDERAÇÕES FINAIS}

O conhecimento do perfil dos pacientes atendidos representa um pilar estratégico para a promoção da organização e aperfeiçoamento dos serviços prestados pela instituição e tem grande utilidade para órgãos de saúde do município, uma vez que os dados epidemiológicos analisados podem ser agregados e consultados para o planejamento de eventuais ações locais.

Conhecer o perfil dos indivíduos que buscam atendimento é, portanto, de fundamental importância para o desenvolvimento de um bom planejamento visando à promoção, prevenção e intervenção na saúde destes usuários.

Com a análise dos prontuários é possível conhecer as doenças mais predominantes, viabilizando a compreensão do processo saúdedoença destes pacientes, contribuindo para um 
atendimento clínico mais eficaz e direcionado às suas demandas.

As informações obtidas tornam possível aprimorar a assistência atualmente prestada e planejar a implantação de novos serviços a fim de satisfazer às demandas locais..

A dor de origem dentária foi a situação clínica que mais motivou a procura por atendimento. Este fato deixa implícita a importância de promover o autocuidado em saúde bucal e educação em saúde, uma vez que a dor, frequentemente é consequência de patologias que podem ser prevenidas.

A interpretação dos dados produzidos neste estudo constitui, portanto, subsídios que podem nortear as futuras ações de intervenção em saúde, de modo a contribuir para a redução da incidência e prevalência de diversos agravos orais na a população atendida.

\section{REFERÊNCIAS}

ANDRIOLA, FO. et al. Perfil sociodemográfico, epidemiológico e comportamental dos pacientes atendidos no ambulatório de exodontia da FO-UFRGS e a efetividade dos atendimentos realizados. Arq Odontol., Belo Horizonte, abr./jun. 2015. [acesso em 04 set. 2016]. 51(2): 104-115. Disponível em: <http://revodonto.bvsalud.org/scielo.php?pid=S151 6-09392015000400006\&script=sci_arttext\&tlng=pt $>$.

BOLAN, V.; MOTTA, MV. Responsabilidade social no ensino superior. Revista de Educação, 2007 [acesso em 03 set 2016]. 10(100): 204-210. Disponível em: <http://pgsskroton.com.br/seer/index.php/educ/article/v iew/2154>. Acesso em: 19 nov. 2016.

BORGES, CM. et al. Perfil das perdas dentárias em adultos segundo o capital social, características demográficas e socioeconômicas. Ciênc. saúde coletiva, Rio de Janeiro, jun. 2014. [acesso em 30 jul 2016]. 19(6): 1849-1858. Disponível em: <http://www.scielo.br/scielo.php?script=sci_arttext\&pi $\mathrm{d}=\mathrm{S} 1413-81232014000601849 \& \operatorname{lng}=\mathrm{en} \& \mathrm{nrm}=\mathrm{iso}$ >.
BRASIL. Ministério da Saúde. Projeto SB Brasil 2003: condições de saúde bucal da população brasileira 20022003: resultados principais. Brasília: Ministério da Saúde, 2004. [acesso em 02 fev 2016]. Disponível em: <http://bvsms.saude.gov.br/bvs/publicacoes/condicoes_ saude_bucal.pdf $>$.

Ministério da Saúde. Projeto SB Brasil 2010: Pesquisa Nacional de Saúde Bucal - Resultados Principais. Brasília: Ministério da Saúde, 2011. [acesso em 29 set 2016]. Disponível em:

<http://dab.saude.gov.br/CNSB/sbbrasil/arquivos/proje to_sb2010_relatorio_final.pdf $>$.

CASTILLO, AGL. et al. Transtornos de ansiedade. Revista Brasileira de Psiquiatria, 2000. [acesso em 14 maio 2016]; 22: 20-23. Disponível em:

$<$ http://www.scielo.br/scielo.php?script=sci_arttext\&pi $\mathrm{d}=\mathrm{S} 1516-44462000000600006>$.

CIMOES, R. et al. Influência da classe social nas razões clínicas das perdas dentárias. Ciênc. saúde coletiva, Rio de Janeiro, dez. 2007 [acesso em 4 out 2016]; 12(60: 1691-1696. Disponível em: <http://www.scielo.br/scielo.php?script=sci_arttext\&pi $\mathrm{d}=\mathrm{S} 1413-81232007000600030 \& \operatorname{lng}=\mathrm{en} \& \mathrm{nrm}=\mathrm{iso}>$

COSTA, SM.; VASCONCELOS, M; ABREU, MHNG. Impacto da cárie dentária na qualidade de vida de adultos residentes no entorno de Belo Horizonte, MG, Brasil. Ciênc. saúde coletiva, Rio de Janeiro, jul. 2013. [acesso em 03 jun 2016]; 18(7) 1971-1980. Disponível em:

<http://www.scielo.br/scielo.php?script=sci_arttext\&pi $\mathrm{d}=\mathrm{S} 1413-81232013000700012 \& \operatorname{lng}=\mathrm{en} \& \mathrm{nrm}=\mathrm{iso}$ >.

DOMINGOS, PSA.; ROSSATO, EM.; BELLINI, A. Levantamento do Perfil Social, Demográfico e Econômico de Pacientes Atendidos na Clínica de Odontologia do Centro Universitário de AraraquaraUniara. ReBraM, 2014. [acesso em 07 mar 2016]; 17(1): 37-50. Disponível em: $<$ http://revistarebram.com/index.php/revistauniara/artic le/view/3>.

FRANÇA, DCC. et al. Perfil epidemiológico dos participantes do Programa de diagnóstico e prevenção do câncer de boca em Mato Grosso. Arq. Odontol., Belo Horizonte, jun. 2011. [acesso em 25 out 2016]; 47(2): 90-94. Disponível em:

<http://revodonto.bvsalud.org/scielo.php?script=sci_art text\&pid=S15169392011000200006\&lng=pt\&nrm=iso

FERREIRA, LL. et al. Odontalgia associada a variáveis socioeconômicas, psicossociais e saúde bucal. Revista Dor, 2012. [acesso em 14 mar 2016]; 13(40: 343-9. Disponível em: <http://repositorio.ufpa.br/jspui/handle/2011/7750>. 
FONSECA, LLV.; NEHMY, RMQ.; MOTA, JAC. O valor social dos dentes e o acesso aos serviços odontológicos. Ciênc. Saúde Coletiva, out. 2015. [acesso em 10 jun 2016]; 20(10):3129-3138. Disponível em: FapUNIFESP (SciELO). http://dx.doi.org/10.1590/1413812320152010.00172015 .

GABARDO, MCL. et al. Social, economic, and behavioral variables associated with oral health-related quality of life among Brazilian adults. Ciencia \& saude coletiva, 2015. [acesso em 07 jan 2017]: 20(5): 15311540. Disponível em:

<http://www.scielo.br/scielo.php?pid=S1413$81232015000501531 \&$ script=sci_arttext\&tlng=es $>$.

GIBILINI, C. et al. Acesso a serviços odontológicos e autopercepção da saúde bucal em adolescentes, adultos e idosos. Arquivos em odontologia, 2010. [acesso em 30 jul 2016]. 46.(4): 213-223. Disponível em: $<$ https://seer.ufmg.br/index.php/arquivosem odontologia/article/view/1844>.

GOMES R.; NASCIMENTO, EF.; ARAÚJO, FC. Por que os homens buscam menos os serviços? As explicações de homens com baixa escolaridade e homens com ensino superior. Cad. saúde pública, 2007. [acesso em 17 mar 2016]; 23(3): 565-574. Disponível em: http://www.scielosp.org/pdf/csp/ v23n3/15.pdf?hc_location=ufi >.

IBGE. Instituto Brasileiro de Geografia e Estatística. Estimativa da População 2016. [acesso em 09 mai 2016]. Disponível em: 〈http://cod.ibge.gov.br/31R>.

MAIA, FBM. et al. Perfil socioeconômico dos usuários e motivo de procura de uma clínica de ensino. Rev Cubana Estomatol, 2016. [acesso em 01 ago 2016]; 53(2): 17-23. Disponível em: <http://scielo.sld.cu/pdf/est/v53n2/est03216.pdf>.

MELO, SMA. et al. Avaliação do perfil periodontal dos pacientes atendidos na faculdade de Odontologia da UniEvangélica. Braz J Periodontol, june, 2016. [acesso em 10 fev 2017]; 26(02). Disponível em: <http://revistasobrape.com.br/arquivos/2016/junho/rev perio_junho_2016_publ_site_pag-07_a_13.pdf $>$.

NASCIMENTO, S. et al. Condições dentárias entre adultos brasileiros de 1986 a 2010. Rev. Saude Publica, dez. 2013. [acesso em 10 nov 2017]; 47: 69-77. Disponível em:

<http://www.journals.usp.br/rsp/article/view/76755>.

NICO, LS. et al. Saúde Bucal autorreferida da população adulta brasileira: resultados da Pesquisa Nacional de Saúde 2013. Ciênc. Saúde Coletiva, fev. 2016. [acesso em 30 jul 2016]: 21(2):389-398.

Disponível em: <http://www.scielo.br/pdf/csc/v21n2/1413-8123-csc21-02-0389.pdf $>$.

OLIVEIRA, MRG. et al. RPS (Registro Periodontal Simplificado): método rápido e simples na identificação precoce da doença periodontal. Odontologia Clínico-Científica (Online), 2015. [acesso em 18 abr 2017]; 14(1): 554-558. Disponível em: <http://revodonto.bvsalud.org/scielo.php?script=sci_art text\&pid=S1677-38882015000100002>.

PEREIRA, SM. et al. Extensão universitária e trabalho voluntário na formação do acadêmico em Odontologia. Arquivos em Odontologia, 2011. [acesso em 28 mai 2017]; 47(2): 95-103. Disponível em: <http://revodonto.bvsalud.org/scielo.php?pid=S151609392011000200007\&script=sci_arttext $>$.

RONCALLI, AG. Epidemiologia e saúde bucal coletiva: um caminhar compartilhado. Ciênc. saúde coletiva, Rio de Janeiro, mar. 2006. [acesso em 09 mai 2016]: 11(1): 105-114. Disponível em:

<http://www.scielo.br/scielo.php?script=sci_arttext\&pi $\mathrm{d}=\mathrm{S} 1413-81232006000100018 \& \operatorname{lng}=\mathrm{en} \& \mathrm{nrm}=\mathrm{iso}>$.

SANCHEZ, HF.; DRUMOND, MM. Atendimento de urgências em uma Faculdade de Odontologia de Minas Gerais: perfil do paciente e resolutividade. RGO. Revista Gaúcha de Odontologia, 2011. [acesso em 19 dez 2017]: 59(1): 79-86. Disponível em: $<$ http://revodonto.bvsalud.org/scielo.php?pid=S1981$86372011000100012 \&$ script=sci_arttext $>$.

SILVA, CB. et al. Frequência das disfunções temporomandibulares (DTM) e sua relação com a ansiedade e a depressão entre usuários que procuram o setor de odontologia em uma unidade de saúde. Rev. APS, 2014. [acesso em $29 \mathrm{dez}$ 2017]: 17(4). Disponível em: $<$ https://aps.ufjf.emnuvens.com.br/aps/article/view/192 $0>$.

SPEZZIA, S.; CALVOSO JÚNIOR, R. Atendimento Odontológico em Hipertensos. Journal of Health Sciences, 2017. [acesso em 19 jan 2017]: 19(1): 43-46. Disponível em:

<http://www.pgsskroton.com.br/seer/index.php/JHealth Sci/article/viewFile/3991/3514>.

Bianca dos Santos Silva

Odontóloga pela Faculdade Maria Milza - FAMAM

Roberto Carlos de Carvalho Cerqueira

Graduando de Odontologia Faculdade Maria Milza FAMAM 
Larissa Rolim Borges-Paluch

Doutora em Ciências Biológicas - UFPR. Docente do

Mestrado em Desenvolvimento Regional e Meio

Ambiente da FAMAM.

Cláudia Cecília Blaszkowski de Jacobi

Doutora em Ciências - USP, Mestre em Linguística Aplicada e Estudos da Linguagem pela Pontifícia Universidade Católica de São Paulo. Docente do

Mestrado em Desenvolvimento Regional e Meio Ambiente da FAMAM e Editora da Revista Textura. 